Pure and Applied Mathematics Quarterly

Volume 10, Number 2

(Special Issue: In memory of

Andrey Todorov, Part 3 of 3 )

$355-366,2014$

\title{
Zeta Functions for Three-Dimensional Bodies and Lattice Point Problems in the Plane
}

\author{
Jonathan Weitsman \\ Dedicated to the memory of Andrey Todorov
}

\begin{abstract}
We compute the asymptotic series for generating functions for lattice point problems in the plane. These generating functions are zeta functions associated to bodies in Euclidean space. Our tools are elementary. Keywords: convex polytope, Euler Maclaurin formula.
\end{abstract}

\section{InTRODUCTION}

The Gauss circle problem and the Dirichlet divisor problem are classical questions concerning the number of lattice points in a convex body in the plane (see e.g. [6]). Let $R$ be an integer and let $\nu(R)$ denote the number of lattice points in the disc of radius $\sqrt{R}$. Then $\nu(R) \sim \pi R+O\left(R^{\alpha}\right)$, where the conjectured value of $\alpha$ is $1 / 4+\epsilon$. The best known value of $\alpha$ is a little less than $1 / 3$. Similarly, let $\Delta(t)$ denote the number of lattice points in the hyperbolic triangle $\left\{(x, y) \in R^{2}: x \geq 1, y \geq 1, x y \leq t\right\}$. Then $\Delta(t) \sim t \log t+(2 \gamma-1) t+O\left(t^{\alpha}\right)$, where a similar conjecture exists and a similar estimate holds for the exponent $\alpha$.

The purpose of this note is to compute the asymptotic series of the generating functions for the quantities $\nu(R)$ and $\Delta(t)$. These generating functions can be viewed as weighted lattice point counts in bodies in $R^{3}$ : in the case of $\nu$ the

Received December 24, 2012.

Supported in part by NSF grant DMS 12-11819. 
body is a solid paraboloid, while for $\Delta$ it is a body bounded by two planes and a hyperboloid. Remarkably all terms in the asymptotic series for the generating functions are computable using the classical Euler-Maclaurin formula. The asymptotic series for $\nu$ has only one term beyond the one arising from the leading term $\pi R$; that for $\Delta$ has infinitely many terms. We do not know of any way in which these asymptotic series are relevant to the computation of the exponent $\alpha$.

The key tool we use is the classical Euler-Maclaurin formula

$$
\begin{aligned}
\sum_{n=a}^{b} f(n)=\int_{a}^{b} f(x) d x+\sum_{i=1}^{N} \frac{b_{i}}{i !}\left(f^{(i-1)}(b)-f^{(i-1)}(a)\right) & \\
& +(-1)^{N-1} \int_{a}^{b} \frac{B_{N}(\{x\})}{N !} f^{(N)}(x) d x
\end{aligned}
$$

where $b_{i}$ is the $i$-th Bernoulli number and $B_{n}(x)$ is the $n$-th Bernoulli polynomial; see e.g. [1]. Essentially all our estimates are repeated applications of this formula. There has been extensive work during the past two decades on generalizations of this formula to expressions giving the sum of the values of a function over the lattice points in a convex body in arbitrary dimension [7, 3, 4, 2, 8]. Although our sums involve such bodies, we make no use of those results and use only the classical, one dimensional, Euler-Maclaurin formula.

\section{A variant of the Zeta function}

Let $R e s>1$, let $u$ be a positive integer, and define

$$
H(s ; u):=\sum_{n=u}^{\infty} \frac{1}{n^{s}} .
$$

The function $H(s ; u)$ is a close relative of the zeta function, and we can study it using the Euler-Maclaurin formula. Thus

$$
H(s ; u)=\int_{u}^{\infty} \frac{d x}{x^{s}}-\left.\sum_{k=1}^{N}\left(b_{k} / k !\right) D_{x}^{k-1} x^{-s}\right|_{x=u}+R_{N}(s ; u)
$$

where

$$
R_{N}(s ; u)=(-1)^{N} \int_{u}^{\infty}\left(B_{N}\{x\} / N !\right) s(s+1) \cdots(s+N-1) \frac{1}{x^{N+s}} d x
$$

so that 


$$
H(s ; u)=\frac{1}{s-1} \frac{1}{u^{s-1}}-\left.\sum_{k=1}^{N}\left(b_{k} / k !\right) D_{x}^{k-1} x^{-s}\right|_{x=u}+R_{N}(s ; u) .
$$

As a function of $s$ this function is meromorphic for $\operatorname{Re} s>-N+1$ for all $N$, with a simple pole at 1 as for the zeta function. It is therefore meromorphic on the whole plane with a simple pole at 1 . Note that we can extend the definition of $R_{N}(s ; u)$ and $H(s ; u)$ to all $u>0$ by using equations $(2.1)$ and $(2.2)$.

\section{The Zeta function of a Paraboloid}

Let $C$ be the solid paraboloid in $R^{3}$; that is,

$$
C:=\left\{(x, y, z) \in R^{3}: x^{2}+y^{2} \leq z\right\} .
$$

We define the zeta function of the paraboloid (for Re $s$ large) by

$$
Z(s):=\sum_{(x, y, z) \in C \cap Z^{3}} \frac{1}{(1+z)^{s}} .
$$

For any $R \geq 0$, let $\nu(R)$ denote the number of integral points in the closed disc $\left\{(x, y): x^{2}+y^{2}=R\right\}$ of radius-squared $R$. Then for Re $s$ large, we can sum over the horizonal discs and the sum up those discs to get

$$
Z(s)=\sum_{R=0}^{\infty} \frac{\nu(R)}{(R+1)^{s}} .
$$

Our purpose is to study the behavior of $Z(s)$ in the complex plane. We do this by "Euler Maclaurin for the paraboloid $C$ ".

By resumming vertically over the paraboloid instead of horizontally, we see that

$$
Z(s)=\sum_{(x, y) \in Z^{2}} H\left(s ; x^{2}+y^{2}+1\right)
$$


A slight variant of the Euler-Maclaurin formula is the following. Let $\sum_{n \in[a, b]}^{\prime} f(n):=\frac{1}{2} f(a)+f(a+1)+\cdots+f(b-1)+\frac{1}{2} f(b)$; similarly, for $O=[a, b] \times[c, d] \subset R^{2}$, let $\sum_{O}^{\prime} f(x, y):=\sum_{m \in[a, b]}^{\prime} \sum_{n \in[c, d]}^{\prime} f(m, n)$. Then

$$
\begin{aligned}
\sum_{n \in[a, b]}^{\prime} f(n)=\int_{a}^{b} f(x) d x+\sum_{i=2}^{N} \frac{b_{i}}{i !}\left(f^{(i-1)}(b)-f^{(i-1)}(a)\right) & \\
& +(-1)^{N-1} \int_{a}^{b} \frac{B_{N}(\{x\})}{N !} f^{(N)}(x) d x
\end{aligned}
$$

Let $Q$ denote the first quadrant in the plane. Then, using our notation,

$$
Z(s)=4 \sum_{Q}^{\prime} H\left(s ; x^{2}+y^{2}+1\right) .
$$

Now we apply Euler-Maclaurin, first in the $x$ direction and then in the $y$ direction. Since $H\left(s ; x^{2}+y^{2}\right)$ is an even function of both $x$ and $y$, and since we are using the weighted sum, which gives rise to an even differential operator, all terms in the Euler-Maclaurin formula involving derivatives of $H$, except for the remainder term, vanish identically. So we get

$$
Z(s)=4 \int_{Q} H\left(s ; x^{2}+y^{2}+1\right) d x d y+4 r_{m}(s)
$$

where

$$
r_{m}(s)=\int_{Q} \frac{B_{m}\{x\} B_{m}\{y\}}{(m !)^{2}} D_{x}^{m} D_{y}^{m} H\left(s ; x^{2}+y^{2}+1\right) d x d y .
$$

Here is a fact about integrals. Let $f$ be a rapidly decaying function. Then

$$
\int_{Q} f\left(x^{2}+y^{2}+1\right) d x d y=\frac{\pi}{4} \int_{0}^{\infty} f\left(r^{2}+1\right) r d r=\frac{\pi}{4} \int_{1}^{\infty} f(\xi) d \xi
$$

So

$$
Z(s)=\pi \int_{1}^{\infty} H(s ; u) d u+4 r_{m}(s) .
$$

We now use our expression for $H(s ; u)$. We get 


$$
\begin{gathered}
Z(s)=\pi\left[\int_{1}^{\infty} \frac{1}{s-1} \frac{1}{u^{s-1}} d u-b_{1} \int_{1}^{\infty} \frac{1}{u^{s}} d u\right. \\
\left.-\left.\sum_{k=2}^{N}\left(b_{k} / k !\right) D_{x}^{k-2} x^{-s}\right|_{x=1}+\int_{1}^{\infty} R_{N}(s ; u) d u\right]+4 r_{m}(s) .
\end{gathered}
$$

In the next section we will prove the following results.

Lemma 3.3. The remainder term $\int_{1}^{\infty} R_{N}(s ; u) d u$ is analytic for all $s$ with Re $s>$ $-N+2$.

Lemma 3.4. The remainder term $r_{m}(s)$ is analytic for all $s$ with Re $s>-m+2$.

Therefore, taking $m=N$, and since since $-\left.\sum_{k=2}^{N}\left(b_{k} / k !\right) D_{x}^{k-2} x^{-s}\right|_{x=1}$ is analytic in the whole plane, it follows that

$$
Z(s)=\pi\left(\frac{1}{(s-1)(s-2)}-\frac{1}{2 s}\right)+M(s)
$$

where $M(s)$ is analytic for $\operatorname{Re} s>-N+2$.

Taking $N$ large, we see that $M(s)$ is actually analytic on the whole plane. This is the same trick as for $\zeta$ and $H(s ; u)$. So

$$
Z(s)=\pi\left(\frac{1}{s-2}-\frac{1}{s-1}-\frac{1}{2 s}\right)+\text { entire. }
$$

Now let us recall the zeta function satisfies $\zeta(s)=\frac{1}{s-1}+$ entire. Therefore

$$
F(s)=Z(s)-\pi(\zeta(s-1)-\zeta(s)-(1 / 2) \zeta(s+1))
$$

is an entire function.

Comparing with equation (3.1), and using the definition of the zeta function

$$
\zeta(s)=\sum_{n=1}^{\infty} \frac{1}{n^{s}}
$$

we see that 
Theorem 1. The sum

$$
F(s)=\sum_{n=1}^{\infty} \frac{\nu(n-1)-\pi n+\pi+\frac{\pi}{2 n}}{n^{s}}
$$

is a convergent series for Re $s>>0$ which analytically continues to an entire function.

Incidentally we note that if we replace the sum in the definition of $Z(s)$ with a weighted sum (weighting lattice points on the boundary of the paraboloid by $\frac{1}{2}$ their weight in the sum giving rise to $\left.Z(s)\right)$ the term $\frac{\pi}{2 n} n^{-s}$ is absent in (3.5).

\section{Estimates on the REMAinder FOR $Z(s)$.}

To make further progress, we need two preliminary results. Our first Lemma is obvious by elementary calculus.

Lemma 4.1. Let $a, b>0$ be integers and let $f$ be an $(a+b)$-times differentiable function. Then there exist polynomials $P_{q}^{a, b}(x, y)$ such that

$$
D_{x}^{a} D_{y}^{b} f\left(x^{2}+y^{2}+1\right)=\sum_{q=[(a+b) / 2]}^{a+b} P_{q}^{a, b}(x, y) f^{(q)}\left(x^{2}+y^{2}+1\right)
$$

where $\operatorname{deg} P_{q}^{a, b} \leq 2 q-(a+b)$.

Lemma 4.2. (see Bourbaki [1]) The Bernoulli polynomial $B_{m}\{x\}$ is bounded and $(m-1)$-times differentiable. Furthermore,

$$
D_{x}^{k} B_{m}\{x\} / m !=B_{m-k}\{x\} /(m-k) !
$$

for all $k<m$.

We now turn to the main estimates.

Proof of Lemma 3.3. We have

$$
\int_{1}^{\infty} R_{N}(s ; u) d u=F_{N}(s) \int_{1}^{\infty} d u \int_{u}^{\infty}\left(B_{N}\{x\} / N !\right) \frac{1}{x^{N+s}} d x .
$$

where $F_{N}(s)$ is a polynomial.

Since $B_{N}\{x\}$ is bounded, this integral converges if $\operatorname{Re} s>-N+2$. 
Proof of Lemma 3.4. Fix $M>1$ (later we will make $M$ much larger than $2 m$ ). By equation (2.2),

$$
r_{m}(s)=a_{m}(s)-\sum_{k=1}^{M} \frac{b_{k}}{k !} G_{k}(s) e_{m, k}(s)+G_{M+1}(s) c_{m, M}(s)
$$

where

$$
\begin{gathered}
a_{m}(s):=\frac{1}{s-1} \int_{Q} \frac{B_{m}\{x\} B_{m}\{y\}}{(m !)^{2}} D_{x}^{m} D_{y}^{m} \frac{1}{\left(x^{2}+y^{2}+1\right)^{s-1}} d x d y, \\
e_{m, k}(s):=\int_{Q} \frac{B_{m}\{x\} B_{m}\{y\}}{(m !)^{2}} D_{x}^{m} D_{y}^{m} \frac{1}{\left(x^{2}+y^{2}+1\right)^{s+k-1}} d x d y
\end{gathered}
$$

and

$$
c_{m, M}(s):=\int_{Q} \frac{B_{m}\{x\} B_{m}\{y\}}{(m !)^{2}} D_{x}^{m} D_{y}^{m} R_{M}\left(s ; x^{2}+y^{2}+1\right) d x d y
$$

and where

$$
G_{1}(s)=1, G_{k}(s)=(-1)^{k-1} s(s+1) \cdots(s+k-2) \text { for } k \geq 2
$$

are polynomials in $s$.

We first compute $a_{m}(s)$. Applying Lemma 4.1, we have

$$
\begin{aligned}
a_{m}(s)= & \frac{1}{s-1} \int_{Q} \frac{B_{m}\{x\} B_{m}\{y\}}{(m !)^{2}} \sum_{q=m}^{2 m} P_{q}^{m, m}(x, y) \frac{-(s-1) G_{q}(s)}{\left(x^{2}+y^{2}+1\right)^{s+q-1}} d x d y= \\
& -\sum_{q=m}^{2 m} G_{q}(s) \int_{Q} \frac{B_{m}\{x\} B_{m}\{y\}}{(m !)^{2}} \frac{P_{q}^{m, m}(x, y)}{\left(x^{2}+y^{2}+1\right)^{s+q-1}} d x d y .
\end{aligned}
$$

Since deg $P_{q}^{m, m} \leq 2 q-2 m$, and since $B_{m}\{\cdot\}$ is bounded, this integral converges if $\operatorname{Re} s>-m+2$.

Next, we compute $e_{m, k}(s)$. We again apply Lemma 4.1, to obtain 


$$
\begin{aligned}
e_{m, k}(s)=\int_{Q} d x d y \frac{B_{m}\{x\} B_{m}\{y\}}{(m !)^{2}} & \sum_{q=m}^{2 m} P_{q}^{m, m}(x, y) \\
& \times \frac{(-1)^{q}(s+k-1)(s+k) \cdots(s+k+q-2)}{\left(x^{2}+y^{2}+1\right)^{s+k+q-1}}
\end{aligned}
$$

which converges if $\operatorname{Re} s>-m-k+2$.

Last, we compute $c_{m, M}(s)$. We first note that if $M$ is sufficiently large, $R_{M}(s ; \cdot)$ is $2 m$-times differentiable. Then, applying Lemma 4.1

$$
\begin{aligned}
c_{m, M}(s) & =\left.\int_{Q} d x d y \frac{B_{m}\{x\} B_{m}\{y\}}{(m !)^{2}} \sum_{q=m}^{2 m} P_{q}^{m, m}(x, y) D_{u}^{q} R_{M}(s ; u)\right|_{u=x^{2}+y^{2}+1} \\
& =\sum_{q=m}^{2 m} \int_{Q} d x d y \frac{B_{m}\{x\} B_{m}\{y\}}{(m !)^{2}} P_{q}^{m, m}(x, y) \\
& \times \sum_{l=0}^{q-1} \frac{B_{M-q+l+1}\left\{x^{2}+y^{2}+1\right\}}{(M-q+l+1) !} \frac{(-1)^{l+M}(M+s) \cdots(M+s+l-1)}{\left(x^{2}+y^{2}+1\right)^{M+s+l}} .
\end{aligned}
$$

Letting $M$ be sufficiently large, this integral converges for $R e s>-m+2$.

\section{A ZETA FUnCtion FOR A SOLID HYPERBolic OBJECT}

Let $H=\left\{(x, y, z) \in R^{3}: x \geq 1, y \geq 1, z \geq x y\right\}$. We define a zeta function for $H$ by

$$
Y(s):=\sum_{(x, y, z) \in H \cap Z^{3}} \frac{1}{z^{s}} .
$$

This is well defined for Re $s$ large, and if $\Delta(n)$ is the number of integral points in the hyperbolic triangle $\left\{(x, y) \in Z^{2}: x \geq 1, y \geq 1, x y \leq n\right\}$, then

$$
Y(s)=\sum_{n=1}^{\infty} \Delta(n) n^{-s} .
$$


We will see that the singularity structure of $Y$ is more complicated than that for $Z$; the asymptotic expansion of $\Delta(n)$ will have infinitely many terms. Resumming as before, we see that

$$
Y(s)=\sum_{(x, y) \in \tilde{Q} \cap Z^{2}} H(s ; x y) .
$$

where $\tilde{Q}$ is the "shifted quadrant" $\left\{(x, y) \in R^{2}: x \geq 1, y \geq 1\right\}$ in the plane. We apply equation (2.2), namely,

$$
H(s ; u)=\int_{u}^{\infty} \frac{d x}{x^{s}}-\left.\sum_{k=1}^{N}\left(b_{k} / k !\right) D_{x}^{k-1} x^{-s}\right|_{x=u}+R_{N}(s ; u)
$$

so that we get

$$
\begin{aligned}
Y(s) & =\frac{1}{s-1} \sum_{(x, y) \in Z^{2} \cap \tilde{Q}}(x y)^{-(s-1)}-b_{1} \sum_{(x, y) \in Z^{2} \cap \tilde{Q}}(x y)^{-s} \\
& -\sum_{k=2}^{N}\left(b_{k} / k !\right)(-1)^{k-1}(s)(s+1) \cdots(s+k-2) \sum_{(x, y) \in Z^{2} \cap \tilde{Q}}(x y)^{-s-k+1} \\
& +\sum_{(x, y) \in Z^{2} \cap \tilde{Q}} R_{N}(s ; x y) .
\end{aligned}
$$

Thus

$$
\begin{gathered}
Y(s)=\frac{1}{s-1}(\zeta(s-1))^{2}-b_{1}(\zeta(s))^{2} \\
-\sum_{k=2}^{N}\left(b_{k} / k !\right)(-1)^{k-1}(s)(s-1) \cdots(s+k-2)(\zeta(s+k-1))^{2}+\sum_{(x, y) \in Z^{2} \cap \tilde{Q}} R_{N}(s ; x y) .
\end{gathered}
$$

The estimate on the remainder is easier than it was for $Z(s)$. The function $R_{N}(s ; \cdot)$ satisfies $R_{N}(s ; u)=F_{N}(s) V_{N}(s ; u)$, where $F_{N}(s)$ is a polynomial, and

$$
V_{N}(s ; u)=\int_{u}^{\infty}\left(B_{N}\{x\} / N !\right) \frac{1}{x^{N+s}} d x .
$$


Now $V_{N}(s ; u)$ is analytic in $s$ if $R e s>-N+1$, and since $B_{N}\{x\}$ is bounded, $V_{N}(s ; u)$ satisfies the bound

$$
\left|V_{N}(s ; u)\right|<C u^{N+R e s-1}
$$

for $u \geq 1$ and $\operatorname{Re} s>-N+1+\epsilon$.

Thus the sum

$$
\sum_{(x, y) \in Z^{2} \cap \tilde{Q}} R_{N}(s ; x y)
$$

is analytic if $\operatorname{Re} s>-N+2$.

We now use the fact that $\zeta(s)=\frac{1}{s-1}+\gamma+(s-1) \Phi(s)$ where $\Phi$ is entire. Thus

$$
(\zeta(s))^{2}=\frac{1}{(s-1)^{2}}+2 \gamma \frac{1}{s-1}+2 \Phi(s)+2 \gamma(s-1) \Phi(s)+\gamma^{2}+(s-1)^{2} \Phi(s)^{2} .
$$

Recalling that $\zeta(0)=-\frac{1}{2}$, we see that $\Phi(0)=\gamma-\frac{1}{2}$; therefore

$$
\begin{gathered}
Y(s)=\frac{1}{(s-1)(s-2)^{2}}+2 \gamma \frac{1}{(s-1)(s-2)}+\frac{2 \gamma-\frac{3}{4}}{s-1} \\
-b_{1}\left(\frac{1}{(s-1)^{2}}+2 \gamma \frac{1}{s-1}\right)+\left(b_{2} / 2\right)\left(\frac{1}{s}+\gamma\right) \\
-\sum_{k=3}^{N}\left(b_{k} / k !\right)(-1)^{k-1}(s)(s-1) \cdots(s+k-3) \frac{1}{(s+k-2)}+J(s),
\end{gathered}
$$

where $J(s)$ is analytic for $R e s>-N+2$. Also note that

$$
\zeta^{\prime}(s)=-\sum_{n=1}^{\infty} \frac{\log n}{n^{s}}=-\frac{1}{(s-1)^{2}}+\text { entire. }
$$

Since $\frac{1}{(s-1)(s-2)^{2}}=1 /(s-2)^{2}+1 /(s-1)-1 /(s-2)$ and $\frac{1}{(s-1)(s-2)}=1 /(s-$ $2)-1 /(s-1)$, we get, by keeping only the first few terms, that

$$
C_{0}(s):=\sum_{n=1}^{\infty}\left(\Delta(n)-n \log n-(2 \gamma-1) n-\frac{1}{4}+b_{1}(\log n+2 \gamma)\right) n^{-s}
$$


is a convergent series for $R e s$ large which extends to an analytic function for Re $s>0$, since the other terms give rise to negative powers of $n$.

With some more work we can obtain the rest of the terms in this series:

$$
\begin{gathered}
-\sum_{k=3}^{N}\left(b_{k} / k !\right)(-1)^{k-1}(s)(s-1) \cdots(s+k-3) \frac{1}{(s+k-2)}= \\
-\sum_{k=3}^{N}\left(b_{k} / k !\right)(-1)^{k-1}(-1)^{k-2}(k-2) ! \frac{1}{(s+k-2)}+\text { entire }= \\
\sum_{k=3}^{N} \frac{b_{k}}{k(k-1)} \zeta(s+k-1)+\text { entire. }
\end{gathered}
$$

A check shows that this formula also works for the $b_{2}$ term, so that

Theorem 2. The sum

$C_{N-1}(s):=\sum_{n=1}^{\infty}\left(\Delta(n)-n \log n-(2 \gamma-1) n+\frac{1}{2} \log n+\gamma-\frac{1}{4}-\sum_{k=2}^{N} \frac{b_{k}}{k(k-1)} \frac{1}{n^{k-1}}\right) n^{-s}$

is a convergent series for Re $s>>0$ which extends to an analytic function for Re $s>-N+1$.

\section{REFERENCES}

[1] N. Bourbaki, Functions d'Une Variable Réele, Chapitre VI: Développments Tayloriens Géneralisés, Formule Summatoire d'Euler-Maclaurin (1951)

[2] M. Brion and M. Vergne, Lattice points in simple polytopes, Jour. Amer. Math. Soc. 10 (1997), 371-392.

[3] S. E. Cappell and J. L. Shaneson, Genera of algebraic varieties and counting lattice points, Bull. A. M. S. 30 (1994), 62-69.

-S. E. Cappell and J. L. Shaneson, Euler-Maclaurin expansions for lattices above dimension one, C. R. Acad. Sci. Paris Sr. I Math. 321 (1995), 885-890.

[4] V. Guillemin, Riemann-Roch for toric orbifolds, J. Diff. Geom. 45 (1997), 53-73.

[5] G. H. Hardy, Divergent Series, Second Edition, 1991 (unaltered), Chelsea Pub. Co., New-York, N.Y.

[6] A. Ivic, The Riemann Zeta Function. Theory and Applications. Dover Publications, New York, NY, 2003. 
[7] J. M. Kantor and A. G. Khovanskii, Integral points in convex polyhedra, combinatorial Riemann-Roch and generalized MacLaurin formulae, Inst. Hautes Études Sci. preprint. - J. M. Kantor and A. G. Khovanskii, Une application du théorème de Riemann-Roch combinatoire au polynôme d'Ehrhart des polytopes entiers de $R^{d}$, C. R. Acad. SciParis Sér. I Math. 317 (1993), no. 5, 501-507.

-A. G. Khovanski, Newton polyhedra and toroidal varieties, Func. Anal. Appl. 11 (1977), 289-296.

-A. G. Khovanskii and A. V. Pukhlikov, The Riemann-Roch theorem for integrals and sum of quasipolynomials on virtual polytopes, Algebra i Analiz 4 (1992), 188-216, translation in St. Petersburg Math. J. 4 (1993), 789-812.

[8] Y. Karshon, S. Sternberg, J. Weitsman. Euler MacLaurin with Remainder for a Simple Integral Polytope. math.CO/0307125; Duke Math. J. 130, 401-434 (2005)

- The Euler MacLaurin Formula for Simple Integral Polytopes. Proc. Nat. Acad. Sci. USA 100, 426-433 (2003)

\section{Jonathan Weitsman}

Department of Mathematics

Northeastern University

Boston, MA 02115

E-mail: j.weitsman@neu.edu 